\title{
CAUSAS MAL DEFINIDAS DE MORTE E ÓBITOS SEM ASSISTÊNCIA
}

\author{
Augusto Hasiak Santo
}

Trabalho realizado na Faculdade de Saúde Pública da Universidade de São Paulo, São Paulo, SP

\author{
* Correspondência \\ Rua Pará, 21 - apto. 62 - \\ São Paulo/SP \\ CEP: $01243-020$ \\ Tel.: (11) 3258-4760 \\ Fax: (11) 3259-2612 \\ auhsanto@usp.br
}

\begin{abstract}
RESUMO
OBjetivos. Este trabalho estuda a distribuição dos óbitos por causas mal definidas no Brasil, no ano de 2003, entre as quais identifica a proporção de mortes sem assistência.

Métodos. Os dados provieram do Sistema de Informações Sobre Mortalidade, coordenado pelo Ministério da Saúde. As causas mal definidas de morte compreenderam as incluídas no "Capítulo XVIII - Sintomas, sinais e achados anormais de exames clínicos e de laboratório não classificados em outra parte" da Classificação Estatística Internacional de Doenças e Problemas Relacionados à Saúde, décima revisão, capítulo este no qual a categoria R98 identificava a "morte sem assistência". Resultados. No Brasil, em 2003, a causa básica de 13,3\% dos óbitos foi identificada como mal definida, sendo que as proporções maiores ocorreram nas Regiões Nordeste e Norte. Do total de causas mal definidas no país, 53,3\% corresponderam a mortes sem assistência, proporção esta que superou 70\% nos Estados do Maranhão, Piauí, Rio Grande do Norte, Pernambuco, Bahia, Paráiba e Alagoas.
\end{abstract}

Conclusão. Dada a estrutura descentralizada para o levantamento dos óbitos no país, identifica-se a maior responsabilidade dos municííios e, em seguida, dos Estados para o aprimoramento da qualidade das estatísticas de mortalidade.

Unitermos: Causas mal definidas. Morte sem assistência. Causas múltiplas de morte. Estatísticas de mortalidade. Declaração de óbito.

\section{INTRODUÇÃO}

Dentre os dados sobre mortalidade, as causas de morte se apresentam como fonte das mais importantes para que seja conhecido o estado da saúde de populações. Destaca-se a causa básica, estabelecida para prevenir a morte e definida como "(a) a doença ou lesão que iniciou a cadeia de acontecimentos patológicos que conduziram diretamente à morte, ou (b) as circunstâncias do acidente ou violência que produziram a lesão fatal"'. A qualidade dos dados sobre mortalidade fica comprometida quando uma proporção considerável de causas de morte é classificada como mal definida. Trabalho de revisão sobre o estado geral dos dados de mortalidade enviados à Organização Mundial da Saúde (OMS), pelos seus estados membros, examinou diversos indicadores de qualidade, entre eles, a proporção de mortes classificadas em categorias de causas mal definidas. Este estudo incluiu o Brasil entre os países com dados de qualidade média².

A grande proporção de mortes sem assistência no Brasil, incluída no capítulo das causas mal definidas, originou a proposta para a criação de uma categoria específica para a sua identificação na Classificação Estatística Internacional de Doenças e Problemas Relacionados à Saúde, décima revisão (CID- I0)3. Nos bancos de dados sobre mortalidade, os registros dos óbitos apresentam o campo para arquivar a causa básica de morte. Assim, é possível a contagem das mortes codificadas nesta categoria, por meio da causa básica e independente do preenchimento de outros itens da declaração de óbito relacionados à assistência médica.
O Sistema Nacional de Informações Sobre Mortalidade (SIM) foi criado em novembro de 1975, na primeira reunião nacional sobre sistemas de informação de saúde. Na abertura desta reunião, o ministro da Saúde falava da "necessidade de informações completas e atuais sobre - que acontece no Brasil no campo da saúde", classificando o evento como sendo "um passo concreto para a racionalização das ações de saúde". O relatório final desta reunião destacava como um dos objetivos "desenvolver um subsistema de informação de mortalidade para melhorar a cobertura, qualidade e oportunidade dos dados", e, em médio prazo, "realizar análises e/ou estudos especiais, tendendo a determinar e corrigir o sub-registro e as deficiências de qualidade da informaçãa".".

Este trabalho estuda a distribuição dos óbitos por causas mal definidas no Brasil, no ano de 2003, entre as quais identifica a proporção de mortes sem assistência e os óbitos em que causas bem definidas são mencionadas como associadas de morte.

\section{Métodos}

Os óbitos estudados provieram do banco de dados do Sistema de Informação Sobre Mortalidade (SIM), publicado em CD-ROM pela Secretaria de Vigilância em Saúde do Ministério da Saúde ${ }^{5}$, sendo excluídos os óbitos fetais. Foram selecionados todos os óbitos em que a causa básica de morte estivesse incluída no "Capítulo XVIII - Sintomas, sinais e achados anormais de exames clínicos e de laboratório não classificados em outra parte", compreendendo as categorias entre R00 e R99, ditas "causas mal definidas", na CID-103. A seguir, dentre os óbitos deste capítulo, foram novamente selecionados: a) os óbitos 
codificados como "morte sem assistência", classificados na categoria R98 e b) os óbitos em que, como causas associadas de morte, outras causas informadas estivessem incluídas em quaisquer dos demais capítulos da CID-10, ditas "causas bem definidas"3. Dentre estes últimos óbitos, foram identificados os registros em que problemas de codificação, digitação e/ou processamento tivessem determinado a seleção de uma causa básica mal definida. Para a caracterização da "morte sem assistência" não foi levado em consideração o item 45 do modelo atual da declaração de óbito, referente à "assistência médica".

Os registros do banco de óbitos reproduziam em seus campos os respectivos itens da declaração de óbito em uso no Brasil. Campos auxiliares foram incluídos, entre eles, campo para transformar em string único os correspondentes às linhas (a), (b), (c) e (d) da Parte I e da Parte II do Modelo Internacional de Atestado Médico de Causa de Morte (atestado médico da declaração de óbito). Nas unidades da Federação, para a identificação da causa básica e das causas associadas, respectivamente, os dados sobre as causas de morte foram processados automaticamente pelo programa Seleção de Causa Básica (SCB) ${ }^{6}$, exceto no Estado de São Paulo, em que foi usado o programa Declarações de Óbito de São Paulo (DOSP), que consiste na adaptação do SCB para processamento em lote ${ }^{7}$. As causas de morte usadas no estudo foram aquelas mencionadas originalmente no atestado médico (entity axis codes) ${ }^{8}$, definidas e apresentadas por meio da estrutura e das rubricas da CID-101,3,8.

As variáveis estudadas foram causas básica e associadas de morte, regiões e unidades da Federação de residência do falecido. As variáveis médicas e demográficas foram processadas pelos programas dBASE III Plus, versão I.I (Ashton-Tate Corporation, Estados Unidos), Epi Info, versão 6.04d, (Centers for Disease Control and Prevention, Atlanta, Estados Unidos) e Excel 2000 (Microsoft Corporation, Estados Unidos). O programa Separador de Registros de Mortalidade (SRM DBF) (DATASUS) foi usado para, dentre os óbitos por causas mal definidas, recuperar os registros em que causas associadas de morte bem definidas estivessem mencionadas. A distribuição dos óbitos é descrita por meio de percentagens.

\section{Resultados}

No ano de 2003, cerca de 13,3\% dos óbitos registrados no Brasil tiveram a causa básica de morte classificada no capítulo XVIII da CID10, dita mal definida. A distribuição destas causas variou entre as regiões do país, prevalecendo no Nordeste, com 25,9\%, em cujos Estados encontram-se as maiores proporções, e Norte, com $21,2 \%$. Os menores valores ocorreram nas Regiões Centro-Oeste, com 5,7\%, Sul, com 6,7\%, e Sudeste, com 8,9\%. Observa-se ainda, grande variação entre as unidades da Federação, em que o Estado do Maranhão apresenta 37,4\% e o Estado do Mato Grosso do Sul, com o menor valor, 2,4\% de causas mal definidas. (Tabela I e Figura I)

Óbitos sem assistência. Em todo o país, cerca de 7,1\% dos óbitos tiveram a causa básica atribuída à morte sem assistência. De modo análogo às causas mal definidas, as regiões com as maiores proporções foram Nordeste (I8,9\%) e Norte (13,5\%), ao passo que as menores proporções foram verificadas no Centro-Oeste (I,6\%), Sudeste (2,2\%) e Sul (2,8\%). Valores superiores a 20\% de óbitos sem assistência foram observados nos Estados do Maranhão, Paraíba, Piauí e Rio Grande do Norte, enquanto proporções menores que $1 \%$ ocorreram no Rio de Janeiro, Distrito Federal, Mato Grosso do Sul e Mato Grosso. (Tabela I e Figura I).

Óbitos sem assistência dentre as mortes por causas mal definidas. No Brasil, dentre o total de causas mal definidas, aproximadamente 53,3\% correspondem aos óbitos sem assistência. Novamente, as maiores proporções ocorreram na Região Nordeste (73,2\%), na qual com valores acima de $70 \%$ incluem-se os Estados do Maranhão, Piauí, Rio Grande do Norte, Pernambuco, Bahia, Paraíba e Alagoas. No entanto, proporções relativamente elevadas destes óbitos são observadas nos demais Estados, exceto no Rio de Janeiro e Distrito Federal. (Tabela I e Figura I).

Causas de morte bem definidas mencionadas dentre as mortes por causas mal definidas. No banco de dados sobre mortalidade do Brasil em 2003, dentre as 133.434 mortes em que uma causa mal definida foi identificada como causa básica, verificouse a menção de uma ou mais causas bem definidas em 2.479 registros. Destacam-se a parada cardíaca (146.9), mencionada 1.578 vezes, a hipertensão secundária (I| 5.0-||5.9), ocorrendo em 228 registros, a insuficiência cardíaca não especificada (150.9), vista em 189 óbitos, e os diagnósticos incluídos no agrupamento intitulado "fatores suplementares relacionados com as causas de morbidade e mortalidade classificados em outra parte" (Y90-Y98), informados em 77 registros. Verificou-se a menção, em 76 registros, de causas incluídas equivocadamente como mal definidas em uma versão do SCB, dentre as quais as outras anemias especificadas e a anemia não especificada (D64.8-D64.9), em 46 registros.

\section{Discussão}

$\mathrm{Na}$ área da saúde, as políticas e os programas visando o controle de doenças e mortes devem se basear em informações adequadas e oportunas sobre a natureza e extensão dos problemas observados, seus determinantes e sobre o impacto causado nas populações. Estes programas e políticas são monitorados a fim de avaliar o alcance de suas metas e seus objetivos. Sem dúvida, as estatísticas de mortalidade por idade, sexo e causa de morte se constituem na forma mais usada internacionalmente para atender às necessidades de planejamento e programação em saúde 2,9 . O uso destas estatísticas fundamenta-se em suas propriedades vigorosas, tais como a) cobertura nacional decorrente do registro civil para o enterramento do corpo e disposições legais para sucessão de bens e recebimento de pensões, b) uniformidade de forma e conteúdo da declaração de óbito e dos procedimentos operacionais do SIM por meio da coordenação do Ministério da Saúde, c) padronização internacional de processamento de dados por meio da OMS?.

Entretanto, as estatísticas de mortalidade devem, a par de oportunas, revestir-se de qualidade, no sentido de refletirem o padrão das causas de morte ${ }^{9,10}$. O indicador mais simples desta qualidade, mencionado na literatura, consiste na proporção de mortes atribuídas às causas mal definidas 2,11. Os valores deste indicador refletem a disponibilidade da assistência médica prestada à população e as características do preenchimento da declaração de óbito"'. Esta proporção indica, de 


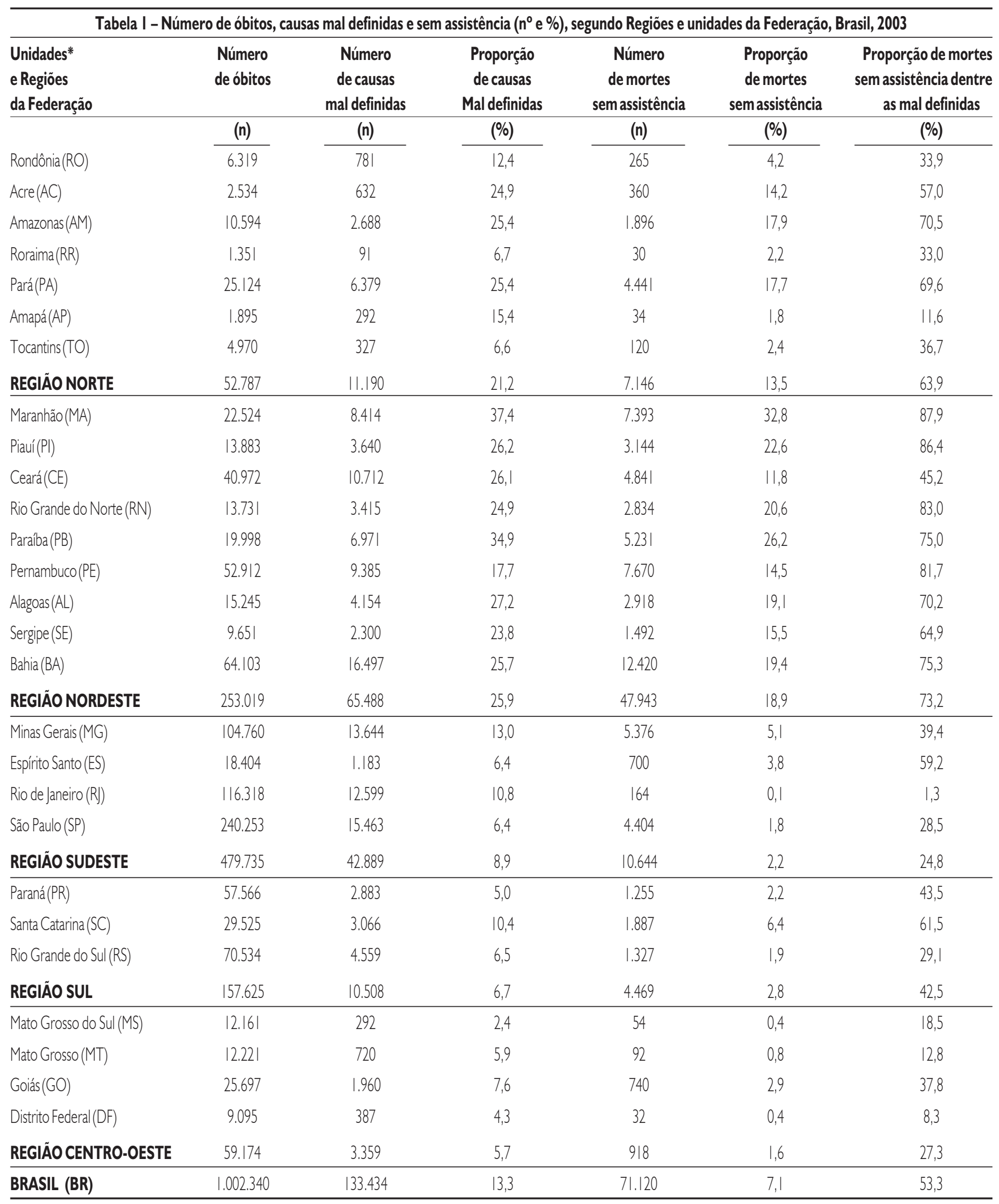

Fonte: Secretaria de Vigilância em Saúde do Ministério da Saúde.

* Entre parênteses siglas das unidades da Federação. 
Figura I - Causas mal definidas de morte, evidenciando mortes sem assistência e outras causas mal definidas, Brasil, 2003

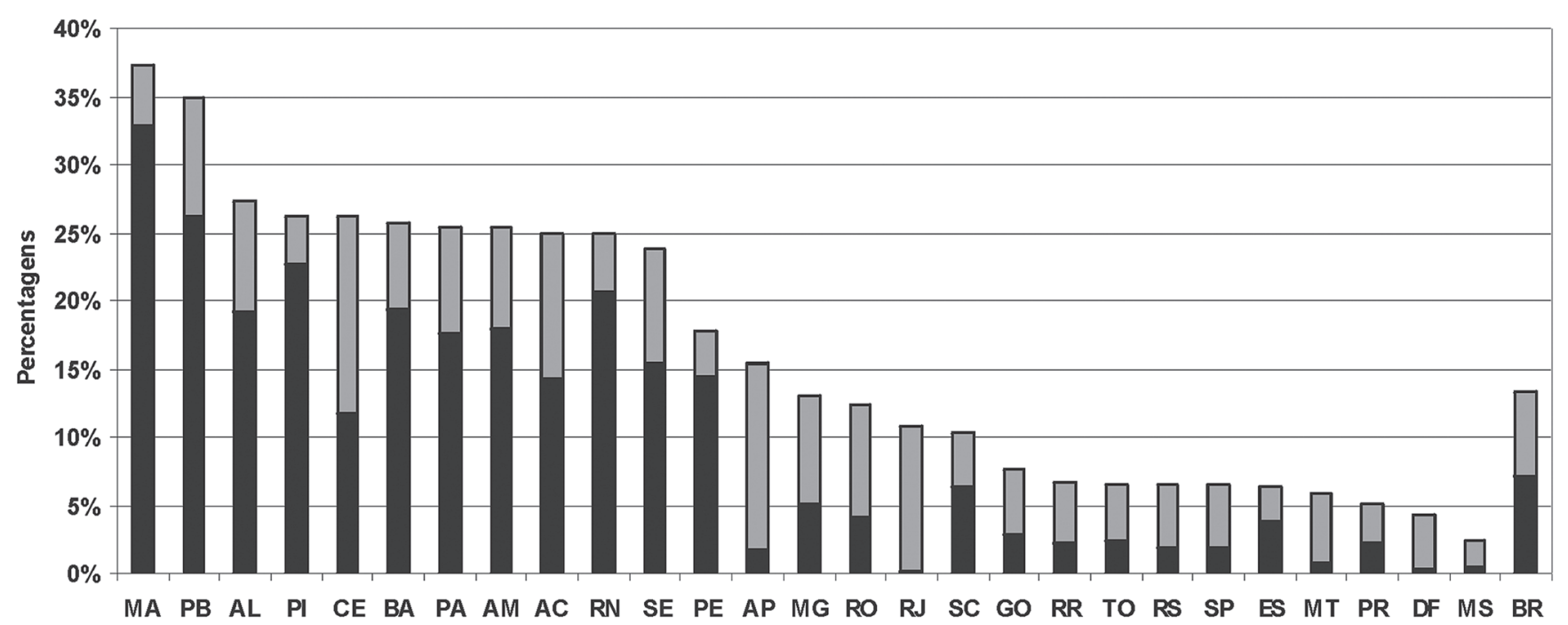

Morte Sem Assistência $\square$ Outras Causas Mal Definidas

modo geral, o cuidado e a consideração durante o preenchimento da declaração de óbito, e pode ser usada como medida aproximada da especificidade dos diagnósticos dados pelo médico atestante?.

Nos anos iniciais da instalação do SIM no país, a proporção de mortes mal definidas manteve-se em torno de 20\%, entre 1979 e 1986. A partir do ano de 1987, inicia-se um declínio progressivo desta proporção, que alcança cerca de $15 \%$ em 1996 e atinge $13,3 \%$ em $2003^{5}$. Analisados estes valores sob a perspectiva da queda, verifica-se um aumento da qualidade das estatísticas de mortalidade. Entretanto, o panorama geral do país encobre um quadro menos favorável, pois engloba as Regiões Nordeste e Norte, respectivamente com cerca de $26 \%$ e $21 \%$ de causas mal definidas, incluindo Estados com proporções acima de 30\%, como Maranhão e Paraíba, e acima de 25\%, como Amazonas, Pará, Piauí, Ceará, Alagoas e Bahia, quadro este que praticamente compromete o valor das causas de morte.

Neste estudo, as mortes por causas mal definidas ficaram restritas àquelas incluídas no capítulo XVIII da CID-10, codificadas entre R00 e R99. Ressalva-se que os 275 óbitos ocorridos em 2003, classificados na categoria R95 (síndrome da morte súbita na infância), embora sejam excluídos dentre as causas mal definidas', foram considerados neste trabalho, a fim de manter comparabilidade com os dados do Ministério da Saúde e de outros estudos na literatura. Por outro lado, os critérios oficiais em vigor, estabelecidos pelo Comitê de Referência da OMS para a Atualização da CID- I0, incluem adicionalmente como causas mal definidas os diagnósticos seguintes: parada cardíaca não especificada (146.9), hipotensão não especificada (195.9), outros transtornos e os não especificados do aparelho circulatório (199), insuficiência respiratória aguda (196.0), insuficiência respiratória não especificada (196.9) e insuficiência respiratória do recém-nascido
(P28.5)'2. Caso estas normas fossem aplicadas no presente estudo, o número das causas mal definidas no país totalizaria | 45.523 óbitos, correspondendo à proporção de $14,5 \%$.

De modo paralelo, é preocupante a observação de que 53,3\% das causas mal definidas sejam constituídas por mortes sem assistência. Esta percentagem observada para o país reflete a importância destes óbitos em praticamente todas as unidades da Federação, especialmente nos Estados da Região Nordeste. Estes óbitos são registrados em cartórios de registro civil, por testemunhas, geralmente familiares do falecido, ou após o fornecimento da declaração de óbito por Serviços de Verificação de Óbitos (SVO) ou por médico que tenha constatado a morte ${ }^{\mid 3,14,15}$. Trabalhos chamam a atenção para a ocorrência de número relativamente alto de óbitos sem assistência ou com atendimento precário, que impossibilitaram a identificaçãa da causa básica de morte, o que pode ser considerado como indicador de acesso e qualidade da assistência médica recebida pela população ${ }^{16}$.

As normas da OMS para a identificação da causa básica destacam que quando a causa selecionada for classificável no capítulo XVIII e estando informada no atestado médico uma causa bem definida, a causa básica deve ser selecionada novamente como se a afecção mal definida não tivesse sido informada'. A procura de causas bem definidas teve a finalidade de verificar a propriedade da identificação de uma causa básica incluída no capítulo das mal definidas. O encontro de problemas de codificação ou processamento sistemáticos de causas de morte ensejaria a formulação de sugestões para a prevenção dos mesmos. Como causas associadas de morte, as menções da parada cardíaca (146.9) e da insuficiência cardíaca não especificada são esperadas e não se constituem em impropriedades. A parada cardíaca tem seu código transformado para R09.2 pelo SCB, a fim de evitar a superestimação de 
doenças do aparelho circulatório nas estatísticas de mortalidade, porém preservando o código original 146.9 como causa associada. A insuficiência cardíaca e a senilidade informadas no mesmo atestado, por disposições das regras de mortalidade da OMS, associam-se para dar origem a insuficiência cardíaca senil como causa básica que está incluída na mesma categoria que a senilidade ${ }^{1,3}$; no registro do banco de dados permanece a insuficiência cardíaca como causa associada. Por outro lado, não se constituem em causas básicas de morte válidas, a hipertensão secundária e os diagnósticos incluídos no agrupamento dos "fatores suplementares relacionados com as causas de morbidade e mortalidade classificados em outra parte (Y90-Y98)"'. O uso destas categorias pode ser caracterizado como erro de codificação. Estes registros seriam passíveis de correção por meio da atribuiçã̃o de um código válido para as causas de morte informadas nas respectivas declarações de óbito. Por fim, a constatação sobre versões inadequadas e desatualizadas do programa SCB estarem sendo usadas em algumas unidades da Federação, determinando a interpretação de anemias como causas mal definidas.

Nos últimos anos, o Ministério da Saúde promove a descentralização das atividades relacionadas ao levantamento de dados sobre mortalidade, deslocando do Estado para o município, a coleta, a codificação e o processamento da declaração de óbito ${ }^{17}$. Como justificativa, são usados os argumentos da pronta disponibilidade de dados de mortalidade pelos órgãos locais de saúde e a perspectiva maior de pesquisar, quando necessário, esclarecimentos complementares junto aos médicos atestantes, principalmente a respeito das causas de morte informadas de modo incompleto, ilegível ou inadequado. No entanto, o preenchimento do quadro de pessoal treinado e qualificado para o desempenho das atividades de codificação e processamento das causas de morte, antes problemático nos Estados, agravou-se agora nos municípios. Relativamente à busca de esclarecimentos, medida que permitiria diminuir a proporção de causas mal definidas, não se verifica a disponibilidade de orientação precisa a respeito, como sob a forma de manuais técnicos, a exemplo do que ocorre em outros países ${ }^{18,19,20}$. A par disso, poucas são as unidades da Federação com possibilidade de supervisionar o trabalho realizado nos municípios. No banco de dados utilizado neste trabalho, encontram-se registros em que códigos de mortes maternas estão incluídos em óbitos de homens (20) e de infantes (36), que se concentraram, respectivamente, 10 e II no Estado de Minas Gerais. À Secretaria de Vigilância Epidemiológica do Ministério da Saúde resta a tarefa de consolidar dados de qualidade comprometida.

\section{Conclusão}

A queda da proporção das causas mal definidas de morte se constitui em uma das principais medidas para o aprimoramento da qualidade das estatísticas de mortalidade no país. Dada a atual estrutura descentralizada para o levantamento dos dados, recai sobre os municípios a maior responsabilidade para alcançar este objetivo. As secretarias municipais de saúde devem ser integradas por técnicos treinados para executar as atividades, visando conseguir maior precisão das causas de morte. A supervisão contínua dos municípios por parte das secretarias estaduais é essencial para o monitoramento de deficiências de estrutura ou procedimentos nos municípios. Nesta etapa, podem ser detectadas inconsistências entre os itens da declaração de óbito e identificados vícios sistemáticos de procedimentos ocorridos nos municípios. Dados confiáveis e precisos serão consolidados no país pelo Ministério da Saúde.

\section{Conflito de interesse: não há}

\section{SUMMARY}

\section{ILL-DEFINED CAUSES OF DEATH AND UNATTENDED DEATHS, BraZIL, 2003}

BACKGROUND. We studied the distribution of deaths from ill-defined causes that occurred in Brazil during 2003, from which was identified the proportion of unattended deaths.

METHODS. Data were obtained from the Mortality Information System, coordinated by the Ministry of Health. Causes of death included in "Chapter XVIII - Symptoms, signs and abnormal clinical and laboratory findings, not classified elsewhere" of the International Statistical Classification of Diseases and Related Health Problems, tenth revision, were considered ill-defined, among which the category R98 identified "unattended deaths".

RESULTS. In Brazil during 2003 the underlying causes of I3.3\% of deaths were included in the Chapter ofill-defined causes, and the highestproportions ofthese deathsoccurred inthe Northeastand North Regions. Consideringthe total deaths from ill-defined causes, $53 \%$ correspond to unattended deaths. This proportion increased to over $70 \%$ in the states of Maranhão, Piauí, Rio Grande do Norte, Pernambuco, Bahia, Paraíba and Alagoas.

CONCLUSION. Due to the decentralized structure of data collection in the country, we believe that the municipalities bear the major responsibility, followed by the states, for upgrading the quality of mortality statistics. [Rev Assoc Med Bras 2008; 54(I): 23-8]

KEY WORDS: III-defined causes of death. Unattended death. Multiplecauses-of-death. Mortality statistics. Death certificate.

\section{REFERÊNCIAS}

I. Organização Mundial da Saúde. Classificação estatística internacional de doenças e problemas relacionados à saúde: $10^{a}$ rev. São Paulo: Centro Colaborador da OMS para a Classificação de Doenças em Português/ EDUSP; 1993. v.2.

2. Mathers CD, Fat DM, Inoue M, Rao C, Lopez AD. Counting the dead and what they died from: an assessment of the global status of cause of death data. Bull World Health Organ. 2005;83: 171-7.

3. Organização Mundial da Saúde. Classificação estatística internacional de doenças e problemas relacionados à saúde: $10^{\mathrm{a}}$ rev. São Paulo: Centro Colaborador da OMS para a Classificação de Doenças em Português/ EDUSP; | 993. v. I.

4. Ministério da Saúde. Primeira reunião nacional sobre sistemas de informação de saúde. Brasília (DF): Ministério da Saúde; 1975.

5. Banco de dados dos Sistemas de Informação sobre Mortalidade (SIM) e Nascidos Vivos (SINASC): 1997 a 2003 [CD-ROM]. Ministério da Saúde, Secretaria de Vigilância em Saúde. Brasília (DF): Ministério da Saúde; 2005.

6. Santo $A H$, Pinheiro $C E$. Uso do microcomputador na seleção da causa básica de morte. Bol Oficina Sanit Panam. 1995; 1 19:319-27.

7. Pinheiro CE, Santo AH. Processamento de causas de morte em lote pelo sistema de seleção de causa básica. Rev Saúde Publica. 1998;32:72-3.

8. Santo AH. Causas múltiplas de morte: formas de apresentação e métodos 
de análise [tese]. São Paulo: Faculdade de Saúde Pública, Universidade de São Paulo; 1988.

9. Mortality Statistics Branch, Division of Vital Statistics, National Center for Health Statistics. Mortality data from the National Vital Statistics System. MMWR Morb Mortal Wkly Rep. 1989;38: I 19-23.

I0. D'Amico M, Agozzino E, Biagino A, Simonetti A, Marinelli P. Ill-defined and multiple causes on death certificates. A study of misclassification in mortality statistics. Eur J Epidemiol. 1999; I 5: 141-8.

II. Silvi J. On the estimation of mortality rates for countries of the Americas. Epidemiol. Bull. 2003;24:I-5.

12. Update Reference Committe. World Health Organization. Cumulative official updates to ICD- I O. Includes proposals ratified by $\mathrm{HoCNHO}$ at HoC Meeting in Tokyo; 2005. [cited 2006 mayo 9]. Avaliable from: http:/ /www.who.int/classifications/committees/ICDCombinedUpdates.pdf.

13. Brasil. Lei n. 6.015, de 31 de dezembro de 1973. Dispõe sobre os registros públicos, e dá outras providências. Diário Oficial da União, Brasília (DF).; 1973; 31 dez. [citado out 2007]. Disponível em: http:// www.spu.planejamento.gov.br/conteudo/legislacao/leis/ lei_60I5_31_12_73.htm.

14. Conselho Federal de Medicina. Resolução no 1.779/2005. Regulamenta a responsabilidade médica no fornecimento da declaração de óbito. Diário Oficial da União (DF). 05 dez 2005, Seção I, p. 121 .

15. Lei Estadual no 5.452. Reorganiza os serviços de verificação de Óbitos no Estado de São Paulo. Diário Oficial do Estado de São Paulo; 1986, 23 dez.
16. Faundes A, Parpinelli MA, Cecatti JG. Mortalidade de mulheres em idade fértil em Campinas, São Paulo (1985-1994). Cad Saúde Pública 2000; 16:671-9.

17. Ministério da Saúde. Sistema de informações sobre mortalidade: manual de procedimentos. Brasília (DF): Ministério da Saúde; 1999

18. National Center for Health Statistics, Centers for Disease Control and Prevention, U.S. Department of Health and Human Services. Instruction manual, part 20, ICD-I0 cause-of-death querying, 2006. Hyattsville: National Center for Health Statistics; 2005.

19. Waller G, Watts $S$. Quality activities for morbidity and mortality coding. In: Meeting of Heads of WHO Collaborating Centres for the Classification of Diseases, Brisbane, Queensland, Australia; 2002.

20. Johansson LA. Data quality. In: Proceedings of the International Collaborative Effort on Automating Mortality Statistics; 2003. Washington (DC). Hyattsville: United States of America. U.S. Department of Health and Human Services, Centers for Disease Control and Prevention, National Center for Health Statistics; 2006. v.3.

Artigo recebido: 16/03/07

Aceito para publicação: 21/09/07 